\title{
NEW Ultra Safety Plus Twist
}

Many years before the 2013 Sharps Legislation in Healthcare ${ }^{1}$ was introduced, Septodont had recognised a need, manufactured, introduced and clinically trialled a needle-stick injury safety device - Ultra Safety Plus. With superior quality, bevel indicated needles and a protective sliding sheath, Ultra Safety Plus (USP) ensured that clinical standards were not compromised for the sake of safety and the syringe was adopted by all UK dental schools and many practices over the years.

The British Association of Dental Nurses (BADN) was concerned with the number of needle-stick injuries and sent out a survey to its members in 2014. Of the 1,108 UK dental nurses who responded, over $51 \%$ (567) had received at least one needle-stick injury and of those $1.24 \%$ acquired a blood-borne virus as a result of the injury that equates to seven people! Most injuries took place after injection.

When USP was introduced into a UK dentistry school, ${ }^{2}$ avoidable needle stick injuries reduced from an average of 11.8 to 0 injuries per 1,000,000 hours worked per year and the study concluded that, whilst the cost of safety syringes is comparable to non-disposable syringes, the reduction in cost of management of needle stick injuries, including the psychological effects are significant.

Over two decades on, Septodont have revised the Ultra Safety Plus to make it even simpler to use with a new, patented twist locking device, making it just two steps to secure assembly. The new Ultra Safety Plus Twist is available with either white, single-use, disposable handles or autoclavable blue handles and they still have the high quality Septoject needles that dental practitioners know and trust.

Septodont are proud to be forward thinking and to always have the safety of the dental team and patients at the heart of their business.

Ultra Safety Plus Twist is available now and will replace the old version entirely very shortly. Please act now to avoid any confusion of the two systems. They are not interchangeable.

\section{Changing your practice over to the new Ultra Safety Plus Twist}

1. Do a stock take of all existing Ultra Safety Plus in the practice

2. Move any old stock of Ultra Safety Plus into one designated surgery. Make sure you take all the existing black handles out of

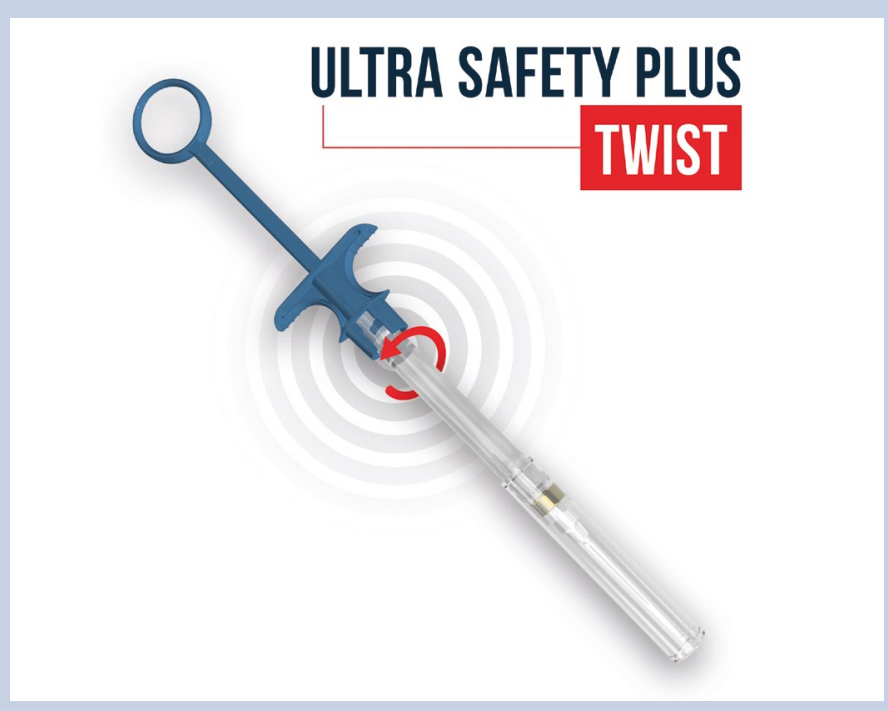

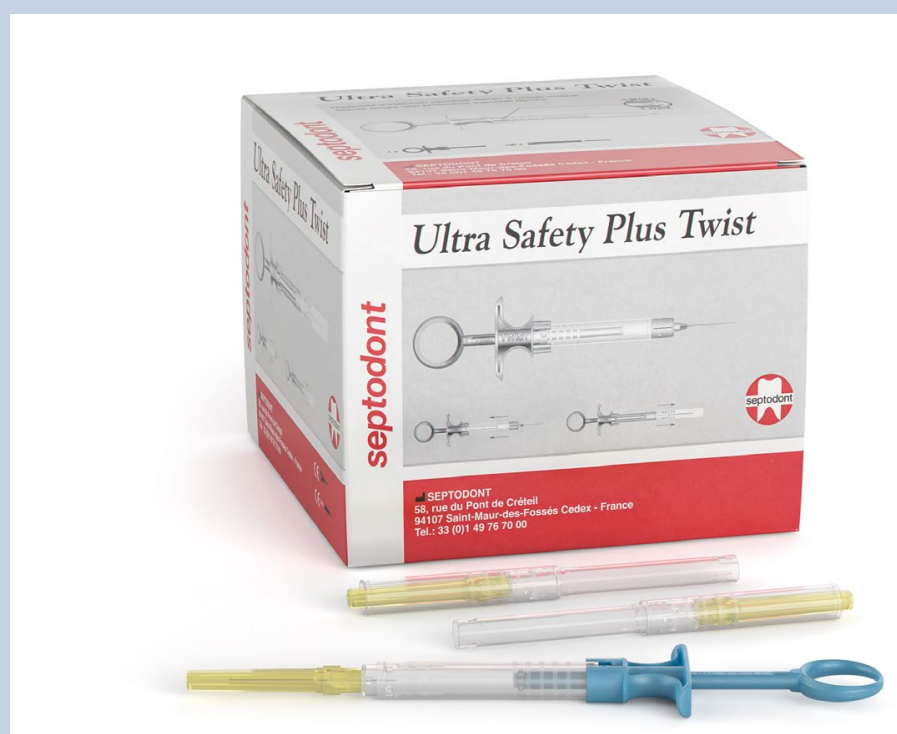

the other surgeries; the black handles are only to be used in the designated surgery. This is important so that you do not have two different systems running simultaneously in the surgeries and you can still use up your existing stocks of Ultra Safety Plus. The two systems are NOT interchangeable. Once you have finished your existing stock of Ultra Safety Plus, please throw the black handles away to eliminate any confusion

3. Please note that the old USP syringe is NOT compatible with the new Twist handles - new syringes are needed as well

4. We recommend purchasing five boxes of Ultra Safety Plus Twist per chair. This will include:

- 500 new USP Twist syringes/needles

- 5 new blue handles - so you can continue with your reprocessing and sterilisation without running out of handles

- Blue handles are available to purchase separately, but are included within every box of syringes

- 25 silicone washers

- Reprocessing and sterilisation protocol

- IFU

- Ensure every member of the clinical team completes the online CPD training with the 'How to Use' video.

The Product Specialists at Septodont are working hard to ensure that customers know about the new Twist device and are on-hand to give advice and on-line training, which can be booked through their main website - www.septodont.co.uk - and the 'How to Use' video is available on YouTube or as part of Septodont's CPD training at www. septodontlearning.co.uk.

\section{References}

1. Health and Safety Executive. Health and Safety (Sharp Instruments in Healthcare) Regulations 2013. Guidance for employers and employees. 2013. Available at: https:// www.hse.gov.uk/pubns/hsis7.htm (accessed May 2021).

2. Zakrzewska J M, Greenwood I, Jackson J. Introducing safety syringes into a UK dental school - a controlled study. Br Dent J 2001; 190: 88-92. 